\title{
TOWARDS THE UTILIZATION OF OPTICAL GROUND-TO-SPACE LINKS FOR LOW EARTH ORBITING SPACECRAFT
}

\author{
Marcus T. Knopp ${ }^{\mathrm{a} *}$, Andreas Spörl ${ }^{\mathrm{a}}$, Marcin Gnat ${ }^{\mathrm{a}}$, Gregor Rossmanith ${ }^{\mathrm{a}}$, Felix Huber ${ }^{\mathrm{a}}$, \\ Christian Fuchs ${ }^{\mathrm{b}}$ and Dirk Giggenbach ${ }^{\mathrm{b}}$
}

\author{
a Space Operations and Astronaut Training, German Aerospace Center (DLR e.V.), Muenchener Str. 20, D-82234 \\ Wessling, Germany \\ ${ }^{\mathrm{b}}$ Institute for Communications and Navigation, German Aerospace Center (DLR e.V.), Muenchener Str. 20, D- \\ 82234 Wessling, Germany \\ * Corresponding Author: marcus.knopp(at)dlr.de
}

\begin{abstract}
The microwave spectrum has become a highly limited resource in satellite communications owing to an ever increasing demand for bandwidth and capacity. Therefore, a shift to the exploitation of optical carrier frequencies is currently underway. Focusing on high-rate transmissions of payload data from remote sensing satellites, operational systems, like the well-known European Data Relay Satellite system, are based on optical inter-satellite links. Besides, direct-to-earth free-space optical communications from low Earth orbiting spacecraft hold high potential for upcoming space missions through lower complexity. In that regard, we study the viability of the ground-to-space beacon laser signal of optical ground stations to be additionally modulated with tele-command tokens. Such an optical return channel could be variously put into use, e.g. to trigger automatic repeat requests of payload data downlinks, for jamming-free control of the spacecraft or for high-rate software uploads to its on-board processor. A particular challenge is posed by the unequal fading behavior of the optical channel regarding the down- and uplinks, which cover asymmetric optical pathways through the atmosphere.

We define the end-to-end architecture of the communication chain including the transmitter on ground and the spacebased receiver. Special attention is given to compatibility with established space data and system standards. Moreover, we examine the effects on the scheduling of satellite control, resulting from a constrained availability of the optical uplink due to cloud blockages. Our analysis aims at the employment of available space protocols for bidirectional optical communications with low earth orbiting spacecraft. Further on, we consider the adoption of upcoming standards to account for the optical fading channel. Certain applications like immediate automatic-repeatrequests for the downlink will require novel, optimized protocols.
\end{abstract}

Keywords: Free-space optical communication, satellite communications, optical ground-to-space links, direct-toearth links, ARQ, TM/TC

\author{
Acronyms/Abbreviations \\ Acknowledgment (ACK), \\ Automated Repeat-Request (ARQ), \\ CCSDS Filer Delivery Protocol (CFDP), \\ Command Link Transmission Units (CLTUs), \\ Consultative Committee for Space Data Systems \\ (CCSDS), \\ Delay Tolerant Networking (DTN), \\ Direct-to-Earth (DTE), \\ European Data Relay System (EDRS), \\ Field of View (FoV), \\ Forward Error Correction (FEC), \\ Four-Quadrant Detector (4QD), \\ Free Space Optical (FSO), \\ Geostationary Earth Orbit (GEO), \\ Ground Station (G/S), \\ Interleaving (IL), \\ Internet Protocol (IP), \\ Inter-Satellite Link (ISL),
}

Local Area Network (LAN),

Low Earth Orbit (LEO),

Line of Sight (LoS),

Message Abstraction Layer (MAL),

Modulator-Demodulator (MODEM),

Network Operation Center (NOC),

Optical Ground Station (OGS),

Optical LEO Downlink (OLEODL),

On-Board Computer (OBC),

Packet Utilization Standard (PUS),

Radio Frequency (RF),

Sequence of Events (SoE),

Service Management (SM),

Software (S/W),

Spacecraft (S/C),

Space Link Extension (SLE),

Space Link Protocol (SLP),

Space Operation Center (SOC),

Telemetry and Tele-Command (TM/TC), 
Telemetry, Tracking \& Command (TT\&C),

Transmission Control Protocol (TCP),

Two-Line Element (TLE),

Universal Space Link Protocol (USLP),

User Datagram Protocol (UDP)

\section{Introduction}

In November 2016 the European Data Relay System (EDRS) has officially gone into operation delivering high-rate data repatriation services for space missions in Low Earth Orbits (LEOs) by means of free-space optical (FSO) inter-satellite links (ISLs) to repeater stations in Geostationary Earth Orbit (GEO) [1]. Representing the first commercial endeavor of that kind, EDRS embodies a tipping point in space communications lately shifting towards optical carrier frequencies, as the radio frequency (RF) spectrum has become increasingly crowded. Optical signaling does not create radio noise and is, therefore, not restrained by frequency coordination. But FSO communications provide more unique features [2]: carrier frequencies in optical bands allow for datarates several orders of magnitude higher than can be achieved with microwaves; moreover, the small beam divergence of FSO communication systems favors power efficient, light-weight, low-profile terminals and inherently contains a high level of physical layer security. On the downside, FSO links are limited to free line of sight (LoS) between the two terminals and are easily impeded by environmental barriers such as cloud cover.

In space, typically no such barriers exist, thus, current applications of optical space links focus on inter-satellite communications. Nevertheless, optical signaling between space and ground passing through Earth's atmosphere has been investigated very well for years [3], [4], [5], [6], [7]. High-capacity optical transmissions from LEO spacecraft $(\mathrm{S} / \mathrm{C})$, so called optical LEO downlinks (OLEODLs), employing direct-to-Earth (DTE) free-space laser connections hold excellent potential to become operationally useable by the end of the decade [8].

OLEODLs are typically established in a two-step process: (1) during the initial acquisition wide-beam beacon light is sent between the communication terminals to reveal their relative positions, (2) during handoff, LoS control is transferred to a narrow communications beam on the side of the space terminal enabling high-capacity link closure and precise pointing during passage of the $\mathrm{S} / \mathrm{C}$ above the optical ground station (OGS) [9].

Here, we investigate the utility of an OGS's uplink beacon laser to provide an optical return channel from ground to the S/C (see Table 1). When modulated, this beacon could administer tele-command (TC)
Table 1: Potential applications of optical uplink transmissions in LEO satellite communications.

\begin{tabular}{|c|c|c|}
\hline Application & Characteristics & Data rate \\
\hline $\begin{array}{l}\text { ACKs for the } \\
\text { optical downlink } \\
\text { of payload data }\end{array}$ & $\begin{array}{l}\text { Part of the duplex } \\
\text { link between } \\
\text { payload memory } \\
\text { and G/S }\end{array}$ & " 100-kbps \\
\hline $\begin{array}{l}\text { ACKs for the } \\
\text { optical downlink } \\
\text { of housekeeping } \\
\text { TM }\end{array}$ & $\begin{array}{l}\text { Part of the duplex } \\
\text { link between } S / C \\
\text { and G/S }\end{array}$ & 1-kbps \\
\hline $\begin{array}{l}\text { High-rate S/W } \\
\text { uploads to the } \\
\text { S/C on-board } \\
\text { computer }\end{array}$ & $\begin{array}{l}\text { Simplex only, } \\
\text { possibly secured } \\
\text { by ACKs in a } \\
\text { downlink }\end{array}$ & $\begin{array}{l}\text { 100-kbps } \\
\ldots \\
\text { 1-Mbps }\end{array}$ \\
\hline $\begin{array}{l}\text { Secure TC } \\
\text { uploads }\end{array}$ & $\begin{array}{l}\text { Simplex only, } \\
\text { possibly secured } \\
\text { by ACKs in a } \\
\text { downlink } \\
\end{array}$ & $\sim 100$-kbps \\
\hline $\begin{array}{l}\text { Exchange of } \\
\text { precision ranging } \\
\text { sequences }\end{array}$ & $\begin{array}{l}\text { Duplex, } \\
\text { symmetric link } \\
\text { required }\end{array}$ & $\begin{array}{l}>1-\text { Mbps } \\
\text { for meter- } \\
\text { precision }\end{array}$ \\
\hline
\end{tabular}

functionality at much higher data-rates than currently yielded by RF uplinks, which are typically performed in S-band. This would mitigate the typically short contact times of LEO S/C above an Earth station and facilitate, for instance, complete on-board software (S/W) uploads within a single pass of the S/C. Moreover, security of S/C operations with respect to jamming or spoofing attacks would be significantly increased due to the high physical layer security of FSO communications. In addition, the in-band return channel could serve for improved error-control of the space-to-ground data transmission, e.g. by means of acknowledgments (ACKs) or automatic repeat requests (ARQs). Finally, a modulated uplink beacon could be used in the future for satellite ranging applications potentially providing improved orbit determinations.

The remaining part of this text is structured as follows: in section 2 we specify the communication scenario underlying our analysis, section 3 gives a general view on the boundary conditions regarding the optical return channel, in section 4 a high-level endto-end systems architecture is suggested. In section 5 we relate this architecture to common space data and system standards; there, we also address impacts of FSO uplinks on ground station (G/S) scheduling and mission planning, before section 6 concludes the paper. 


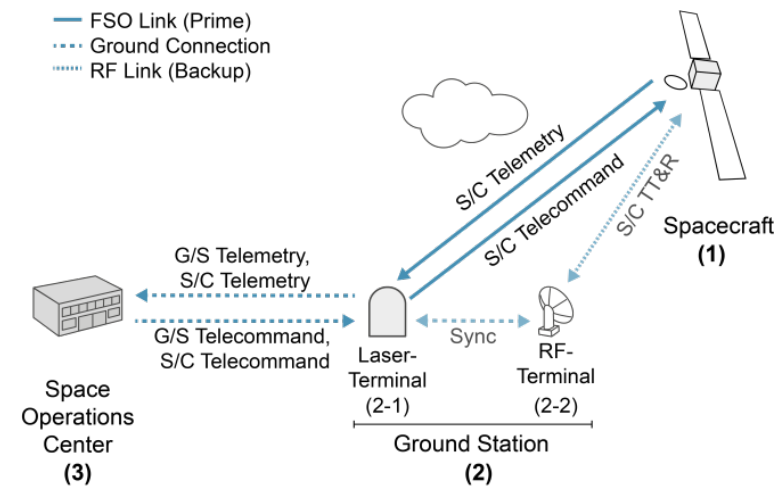

Fig. 1. Elements of the system model considered.

\section{System and Channel Model}

\subsection{System Overview}

We consider a typical space link scenario to conduct telemetry and tele-command (TM/TC) activities.Fig. 1 shows the different elements involved in such a link. Telemetry (TM) signals are transmitted by a spacecraft (1) towards a receiving station on ground (2) and relayed via ground connections to the space operations center (3). TC tokens are transmitted vice versa. Whereas for the time being such links are realized in RF bands, here, we explore the possibility to utilize FSO communications between the S/C and the G/S, particularly the viability of optical uplinks to deliver tele-commands. Local weather conditions at the ground site will largely influence availability of the ground-to-space link (cf. section 3); therefore, a feedback loop between the G/S and the space operations center (SOC) is established to allow for adequate action response. In addition, maintaining RF backup functionality is suggested to mitigate operational availability constraints and ensure continuous serviceability of the S/C.

\subsection{Geometrical Aspects of FSO Space Links}

The data uplink to a typical LEO satellite mission today is performed on-board by semi-hemispheric Sband antennas. Generally, this does not require steering of the antenna field of view (FoV) towards the sending G/S, at least to establish low-rate emergency connections. However, it also allows any antenna inside the visibility footprint of the S/C to direct a data signal into the satellite antenna. Besides general matters of signal interference, jamming or spoofing attacks of uplink transmissions are a major concern in this respect for S/C operators, as in RF bands these can be performed easily and, thus, must be detected and prevented by higher-layer techniques. Capture or blockage of satellite operations is a major thread to any LEO-mission.

In optical uplinks, the on-board data receiver, which is typically in parallel the tracking sensor, i.e. a
Four-Quadrant Detector and Tracking Photo Cell (4QD), has typically a FoV of 10-mrad (or 0.6-deg). Such a small FoV is required to maintain a highprecision tracking accuracy for the downlink beam, as well as to reduce influences of background light onto the 4QD. Optical signal sources outside this FoV-cone will not be detected by the satellite terminal. Interference at this sensor, to be effective, can originate only from a circular area of typically $10-\mathrm{km}$ radius around the eligible OGS, an arrangement that can be easily detected, put the case that the source of interference is able to sustain the necessary pointingrigor, which poses a challenge of its own.

\subsection{FSO Channel Properties}

Optical LEO-to-ground links [10] offer very high throughput of 10-Gbps for e.g. sensor data download, with increasing demand for high data rates to be expected in future systems. These are realized by rather small transmitter terminals on the S/Cs (few cubic decimeters in volume, with a transmitter aperture diameter of only few centimeters) to also comparably small receiver telescopes on ground (typically 40 to $80-\mathrm{cm}$ diameter primary apertures).

The availability of the optical link is influenced by the local cloud cover (cloud-free LoS is required) [8], [11], leading to ground stations to be preferably located in appropriate regions (see Fig. 2). The achievable data rates depend on the link distance (i.e. free-space loss) and the atmospheric transmissivity given by path-integrated aerosol content. Both are influenced by the link elevation. Thus, a variable data rate concept should be implemented through the data format to enable optimum throughput per link session.

The link is further impaired by disturbances through the index-of-refraction turbulence of the free atmosphere, causing self-interference of the coherent laser signal. This effect results in intensity structures (so-called speckles), whose time-dynamics are given by the movement of the air volume by wind and convection; typically the structures vary in the timeframe of milliseconds. Size and strength of these speckles depend on the distance of the laser source from the disturbances and, again, the link elevation [12]. In the downlink (where the disturbing atmosphere is close to the receiver telescope) the speckles will measure only centimeters to decimeters in size, while in the LEO-uplink (where the disturbing atmosphere is far from the receiving end) structures will come to several meters in size. Consequently, in the downlink, speckles-induced fading is easily reduced by larger ground apertures, an effect called aperture averaging [13]. However, in the uplink these speckles are far too large to be compensated by the receiver aperture of the space terminal, and they will influence the signal stability with strong fades and 
surges. Transmitter diversity can reduce the uplink power scintillation index (normalized variance of signal power) by a factor equal to the number of parallel ground transmitters. But additional electronic fading mitigation techniques need to be applied, namely Forward Error Correction (FEC), Interleaving (IL) for spreading of fading-outages, and desirably automated repeat request (ARQ), if an according return channel (here: the downlink) is available.

A physical channel-model based on outages (drop of received power below a certain threshold) can be found through evaluation of the intensity-scintillation index, taking into account both the effect of aperture averaging and transmitter diversity. While the lognormally distributed received power at either side of the optical LEO-GND link is modelled by its equivalent probability density function and spectrum, outages are determined by way of the probability that the received signal intensity falls within a required margin [14].

\section{Boundary Conditions at a Glance \\ 3.1 Required Data Transfer}

FSO data transmissions can only reliably provide ground-to-space connectivity, if they are capable of fulfilling all tasks accomplished by $\mathrm{RF}$ communications today at comparable or a better quality. Communications between spacecraft and ground during routine operations can be categorized in four main tasks: (1) Transmission of tele-commands (TC, sent from ground to space) and (2) reception of telemetry (TM, sent in opposite direction) form the fundamental part of satellite operations. These tasks resemble the day-to-day operations to maintain housekeeping activities. Another two tasks can be considered special versions of those. (3) Reception of payload data is a specialization of TM performed either over the same physical channel as the housekeeping or via a dedicated channel (e.g. by an extra transmitter aboard the S/C). For most LEO satellites such payload data consist of imaging products, but it could also contain results of scientific on-board processes or other measurements. Typically, the amount of payload data exceeds the housekeeping TM by far and is a significant driver for the number of daily ground station contacts of a space mission. (4) S/W uploads to ono-board systems of the S/C, e.g. the on-board computer (OBC) or any sub-system unit, are specializations of TCs.

While the first three tasks are normally used on a daily basis, S/W uploads are only carried out when required. The amount of data to be transferred can vary and depends on the particular platform, the targeted sub-system, and the transfer method (full S/W or just single parts to be updated), but in principle imposes also higher requirements on transmit capacity.

The boundary conditions for all four described tasks strongly depend on the satellite project. The following list is, thus, very generic.

- For housekeeping TM/TC, usually one or two regular ground station contacts per day are sufficient. For the TC-part, it might be even rarer, since command tokens are usually uploaded to an on-board schedule having TCs available for several days in advance. This leads to a drawback in terms of reaction time, though. In general, fewer contacts could also be possible for the TM, but only smaller projects with hard budget constraints tend to allow for really seldom possibilities to verify the S/C status. The data rates needed for housekeeping TM/TC are in general quite low, that is on the order of few kbps.

- For payload data, in contrast, regular downlink possibilities with high data rates are the norm. Several ground station contacts per day are not uncommon. The regularity of these contacts prevents the data to be outdated (a requirement which strongly depends on the mission and might be ignored) and prevents an overflow of the S/C on-board memory. While optical DTE links can give good answers in terms of the transfer rates, the regularity is trickier as outlined in the next section.

- S/W updates are performed irregularly: They can happen several times in only a few weeks, or not at all during a whole year. Depending on the size of the $\mathrm{S} / \mathrm{W}$, these updates can be quite voluminous. Up to several thousand TCs might be needed, which often results in multiple RF ground station passes. This, of course, has a huge impact on operation time and mission cost. In most cases S/W uploads are not time critical. Therefore, for this task optical communications might provide a considerable improvement.

\subsection{Availability Constraints}

Optical ground stations are to be used mainly for very high-speed payload data downlinks from LEO S/C, but could simultaneously offer optical data uplink capability. OGS sites will be located in geographical regions with naturally low cloud cover to maximize data throughput and access to the satellites. Other aspects like site accessibility, presence of infrastructure, terrestrial network connectivity, geographical spread, etc. will further influence site selection. Eventually, up-/downlinks will be performed from a distributed network of optical 


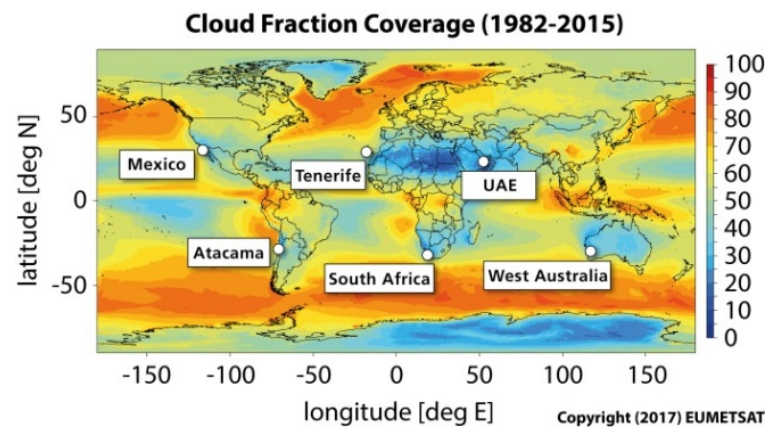

Fig. 2. Potential OGS network with optical down- and uplink capability. Based on [15].

terminals putting up with a high degree of data fragmentation on ground. Fig. 2 presents a first approach for such a network of optical ground stations, as based on current studies [work in progress]. The goal has been to reach global coverage, i.e. enable an optical contact every 1 to 3 orbit(s). This can be achieved by a worldwide OGS-network allowing for seasonal decorrelation of the local climate, which means that winter time and, thus, increased cloud cover on one hemisphere will usually coincide with summer time and, thus, decreased cloud cover on the counter hemisphere. From these perspectives, good OGS sites tend to be located in the arid belts around +/- 23-deg latitude. Polar orbiting satellite missions are geometrically well served by ground stations located near the Earth's poles. However, while some Antarctic areas offer very low cloud probability, the lack of fiber-connections to this continent for the time being prevents their utilization as OGS sites. The same applies to the North of Greenland.
Fractional cloud cover can be read from the color code of Fig. 2. It gives an impression of the mean availability of an OGS site for optical signaling. Detailed long-term cloud cover analyses are necessary to further evaluate the availability of a combination (i.e. network) of OGSs. Recent studies have shown that with around four largely de-correlated OGS sites more than 99-\% link availability can be achieved [16].

\section{End-to-End System Architecture 4.1 Laser Terminals}

Fig. 3 gives an overview of essential elements needed for a typical laser-based communication chain. The ground system (left) consists of an optical aperture, mostly a reflective telescope with a diameter of 40 to $80-\mathrm{cm}$, an optical system (relay assembly, beam splitters, filters a.s.o.) and a receiver frontend, which performs the conversion from the received optical signal to an electrical signal. The processing unit in the ground station performs the decoding of the received data, the coding of the transmitted data, as well as additional tasks, such as providing link status information. In transmit direction, a laser source, usually a low-power seed laser followed by an optical amplifier, generates the optical signal that is transmitted via the optical aperture.

In a space-based laser terminal (right) typically a combined optical aperture is used, which requires splitting between transmit and receive pathways within the optical system (e.g. by optical filters, when using different wavelengths for the up- and downlinks). Again, receiver frontend and laser source are used for reception and transmission, respectively. The FSO modulator-demodulator (MODEM) performs decoding and encoding of the signal streams. (a)

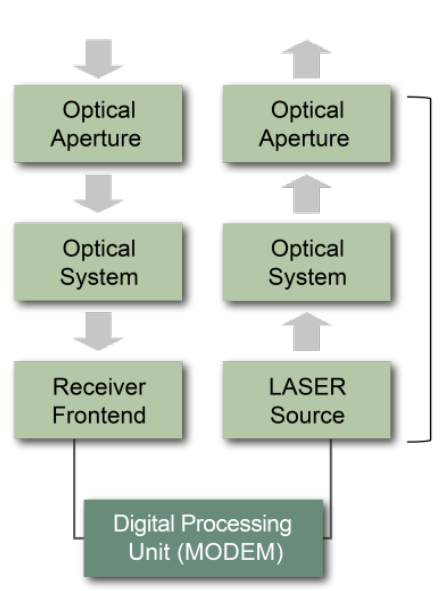

(b)

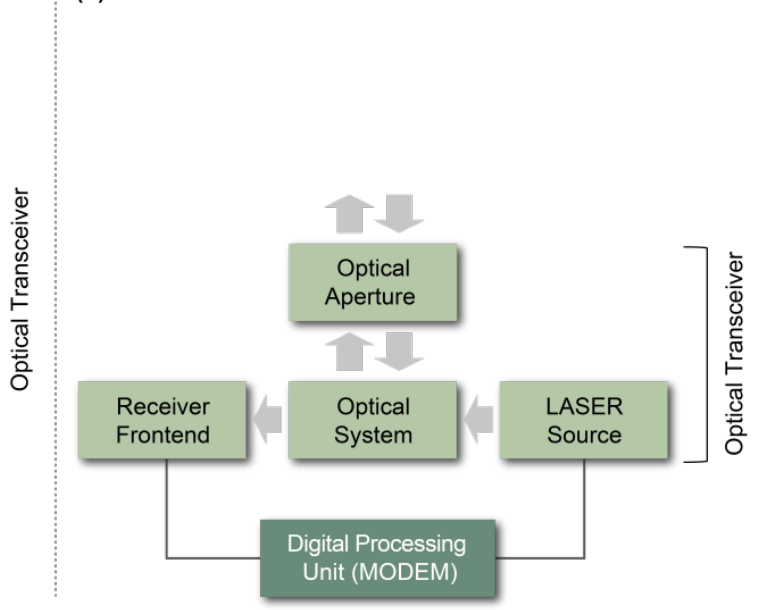

Fig. 3. Overview of essential laser terminal elements. (a) ground terminal at the OGS; (b) space terminal aboard the $S / C$. 


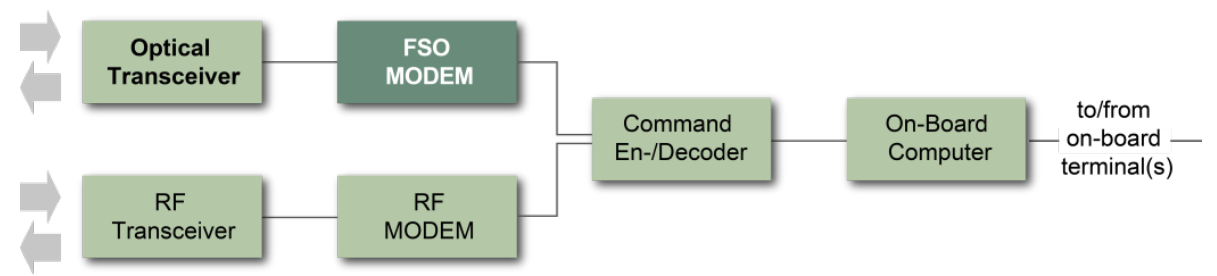

Fig. 4: Overview of essential data handling elements on-board the spacecraft.

\subsection{Spacecraft Transmission System}

A typical RF transmission sub-system aboard a Packet Utilization Standard (PUS) compatible S/C looks like the following (cf. Fig. 4): An RF transceiver forms the air-to-S/C interface, comprised of an antenna, amplifier and up-/downconverter, followed by a MODEM. In the uplink, when a CCSDS conform signal is acquired, this is converted into a bit stream, which is further piped to a command decoding unit. There, the CCSDS frame header is unpacked, checksums are calculated and errors are detected and corrected, if possible. The content of the TC frame is made available to the OBC, which further distributes the command packet to the addressed PUS terminal for execution. At several stages aboard the satellite, ACKs might be requested and reported (eventually to the SOC on ground). This ensures that TCs are executed in the predefined way. This information is downlinked in addition to the housekeeping TM and payload data in the reverse transmission path. For that, either a TM packet is wrapped in a PUS conform way by adding information (e.g. about the on-board terminal that created the packet) to the corresponding frame header, or in case of $\mathrm{AD}$ mode ACKs, so called Command Link Control Words, become part of any of the next TM frames presently available for the downlink. The latter guarantees a fast feedback to enable a continuous uplink stream of TCs.

In case of problems in the up- or downlink chain, manual interaction, i.e. resending failed commands or re-dumping corrupted TM packets, are not uncommon, but also time-consuming. Hence, automation might be implemented to support trouble shooting.

Incorporation of an FSO uplink channel into the on-board Telemetry, Tracking \& Command (TT\&C) sub-system could be performed in the following way:

- By means of an ARQ scheme, it is ascertained that a TC-table can be reliably uploaded from ground to the space terminal.

- TCs are routed to the command decoder, where an ACK scheme similar to the PUS conform data handling, but back-channeled via the optical link, is used to ensure command integrity, to mitigate cases where the ARQ scheme has failed (e.g. due to link outages).
- The command list received is handed over to the satellite OBC as usual, where the commands are added to the command queue.

To avoid potential TC conflicts, e.g. if only parts of commanded instructions have been received on-board, we expect precautionary measures to be developed via dedicated communication protocols customized to the requirements set by the FSO channel.

\subsection{Ground Station}

An FSO-enabled G/S will need some minor modifications from a classical RF station as depicted in Fig. 5: basically a laser terminal needs to be integrated together with few monitoring equipment to keep track of the local weather and potentially of the air traffic above the site.

Equivalent to a classical antenna arrangement, a laser terminal consists of an optical transceiver at the air-to-ground interface and an appropriate digital processing unit handling the electrical data signals (cf. section 4.1). The latter is connected to the local area network (LAN) of the ground site and implements standard networking protocols like Transmission Control Protocol (TCP) or User Datagram Protocol (UDP) to give access to a data stream. For uplinkbackup functionality, transmissions can be directed to an RF terminal by a network switch. Terminals are monitored and controlled by a terminal-server-based sub-system (M\&C Server and GUI clients) that is also handling all processes applicable to the transceivers, i.e. the ground procedures needed to make use of them and the respective data transferred.

To preserve compatibility to global standards and facilitate cross-support between different G/S providers, TM/TC packets are exchanged between G/S and SOC using the CCSDS Space Link Extension (SLE) protocol [17], [18], [19], where the G/S takes the role of the SLE provider and the SOC the role of the SLE user.

\subsection{Space Operations Center}

Routine operations are mainly driven by the mission planning system (MPS). Its main product is a command-ready file containing time-tagged telecommands to execute data-takes, downlinks and all other routine tasks on-board the S/C. This timeline or sequence of events (SoE) is meant to be free of 
conflicts with respect to certain constraints. Amongst them the most prominent are availability restraints on the payload cache memory. Other sub-systems of the SOC take care of TT\&C, flight dynamics products (like orbit determination and propagation) and offline analyses of S/C TM. Fig. 6 shows a simplified sketch of the core elements needed to maintain S/C operations. Taking into account the abovementioned constraints on the availability of an OGS, we anticipate a functional unit within the center's TM/TC sub-system (Dynamic TC Selector) dedicated to process status signals of the $\mathrm{G} / \mathrm{S}$ in real-time.

The ground segment covers, in general, few types of data, which are categorized in Table 2. Real-time data is transferred over several interfaces by different protocols (detailed below) between components in the SOC (e.g. the S/C M\&C system) and the G/S. Essential elements are, here, the communication paths between the sub-systems including the network infrastructure, as well as the respective gateway software (SLE User software). Additionally, requirements on bandwidth, security and reliability aspects need to be addressed.

For offline data, specific file transfer pathways and according mechanisms need to be defined in advance, for example transmission of Two-Line Elements (TLEs) from the Flight Dynamics node to the G/S by automated file transfer. Increasingly, some of these supplies are being provided by means of online services, thus, skipping the necessity of transferring files as such. Instead, only the needed information is delivered on request.

A service management entity (Ground Service Management) is in charge of the offline Management Data related to the ground stations. This includes planning and scheduling of S/C ground-contacts, provision and maintenance of the station configuration for a specific space terminal and reporting on executed passes.

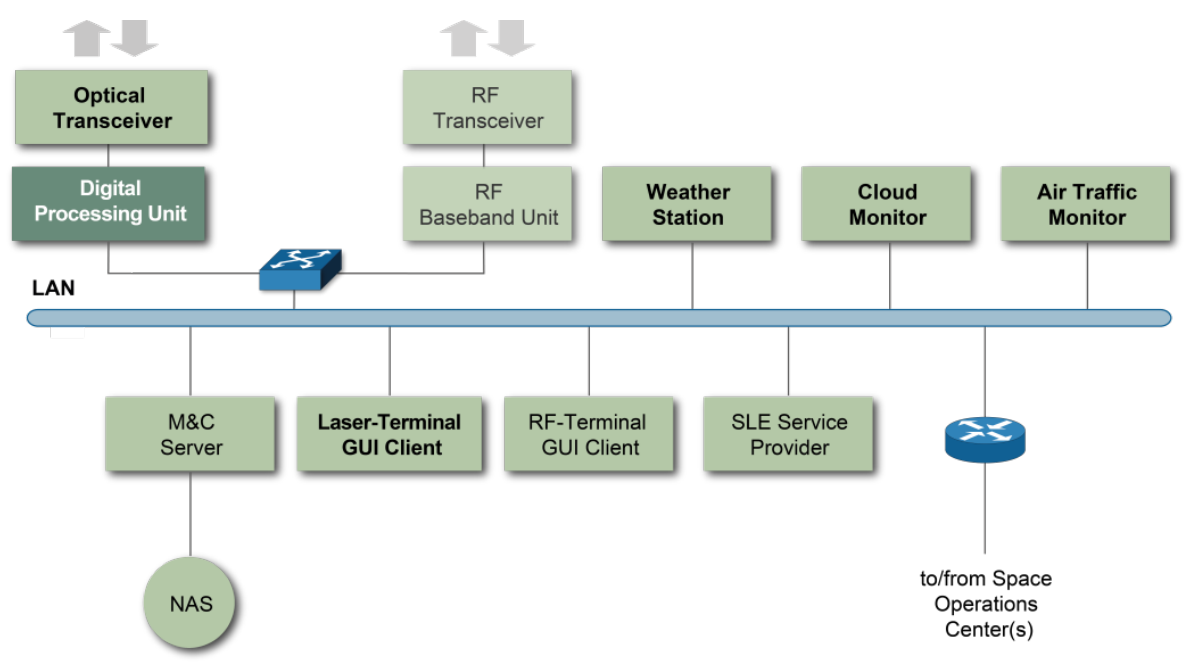

Fig. 5. Overview of essential ground station elements.

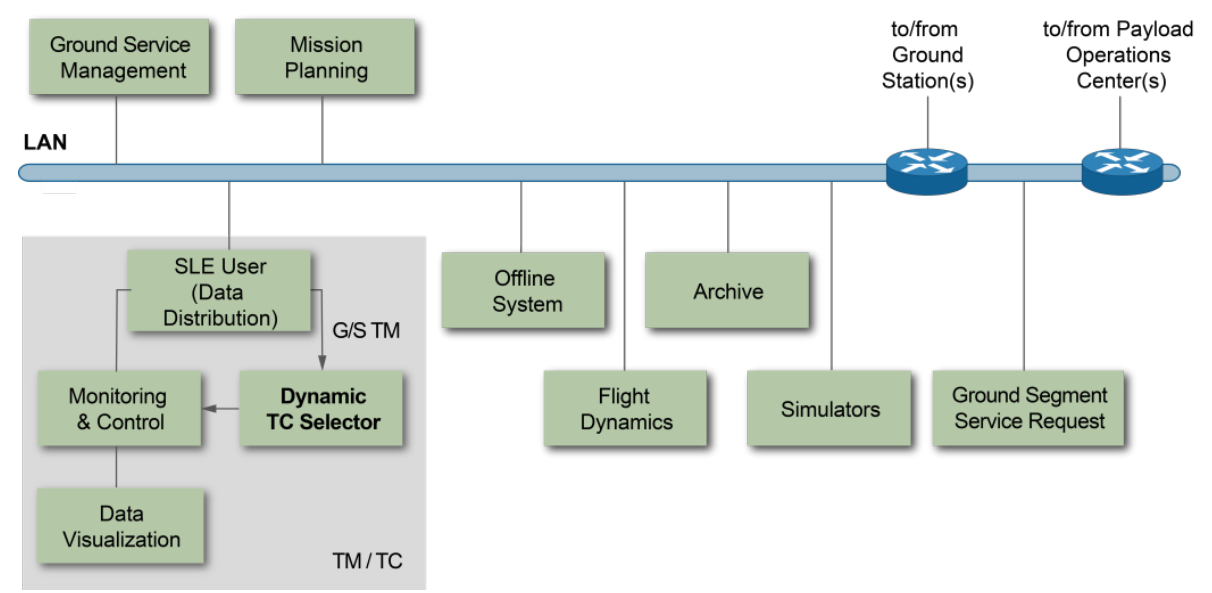

Fig. 6. Overview of essential control center elements. 


\section{Discussion}

\subsection{Space Data and System Standards}

Similar to the table in the previous section, the data transfer protocols can be assigned to different categories. In general, most of the data traffic on ground is handled by Internet Protocol (IP) networks via TCP/IP, sometimes using UDP at the transport layer for specific applications.

Between the control center (SOC) and the G/S connections are typically covered by the SLE protocol. Its implementation builds directly on top of TCP/IP and allows encapsulation of real-time TM/TC in form of Space Link Protocol (SLP) frames or Command Link Transmission Units (CLTUs). Respective services are renowned as Return All Frames, Return Channel Frames and Forward CLTU. The SLE protocol assures space communication specific signaling and data delivery. Both, SLE and SLP, are well established and very mature. Yet, they may impose some challenges with respect to certain applications, such as high-rate uplinks, symmetrical links and demanding real-time operations, which seem to become major domains of FSO space communications.

In future, the Cross Support Transfer Services standard will allow encapsulation of many additional services, which shall extend the capabilities of the communication system on ground [20], [21]. By that, we anticipate handling of optical ground transceivers with their abovementioned peculiarities to become sufficiently supported. For example, the exchange of station TM (weather, clouds, air traffic, a.s.o.) between G/S and SOC (cf. Fig. 1 and Fig. 6) could be covered by this standard. Additionally, the services of Monitoring Data [21] and possibly the Control Service [not yet released] will encompass the provision of direct real-time feedback and control of the ground station status to the control center (SOC or Network Operations Center - NOC).

Table 2: Summary of the data types handled by the ground segment of a space mission.

\begin{tabular}{lll}
\hline & Real-time & Offline \\
\hline \hline Operational & $\begin{array}{l}\text { Payload TM, } \\
\text { S/C House- } \\
\text { keeping TM \& } \\
\text { TC }\end{array}$ & $\begin{array}{l}\text { Payload TM, S/C } \\
\text { history TM, } \\
\text { S/C Ranging, } \\
\text { (Doppler, angle, } \\
\text { etc.) }\end{array}$ \\
\hline Management & Station TM, & Service \\
Data & Station TC & $\begin{array}{l}\text { Management, } \\
\text { Scheduling, } \\
\text { Station } \\
\text { Configuration, } \\
\text { Tracking (Orbit, } \\
\text { TLE) }\end{array}$ \\
\hline
\end{tabular}

The not yet released Universal Space Link Protocol (USLP) shall incorporate further flexibility for space systems to define their TM formats, provide symmetrical links and allow for higher data rates [work in progress]. This may show useful especially for the here discussed FSO links, which intrinsically focus on higher data rates in both up- and down directions.

The transfer of files, as well as internetworking with end-to-end applications, i.e. between S/C subsystems and the respective control units on ground within the control center, are possible by the use of additional standardized protocols, which are encapsulated in either SLP or the Message Abstraction Layer (MAL) guaranteeing their provision within the ground segment. These protocols include CCSDS File Delivery Protocol (CFDP) [22], IP encapsulation [23], [24] and Delay Tolerant Networking (DTN) [25]. Though still under development, the latter is already closely tied to FSO space communications. As soon as space based networks become fully operational (for example with Moon or Mars nodes) large amounts of data are to be transferred. The advantage of high data rates at high power efficiency available via FSO techniques is obvious. But DTN can also help alleviate the abovementioned availability constraints on FSO communications with LEO S/C (see section 3 ), since the respective protocols enable a user to uncouple from the defragmentation process within a distributed OGS- network.

With regards to the data handling on-board the S/C the corresponding CCSDS standard (PUS) allows for more than $50 \%$ overhead in case of small TC packets. Creating this overhead takes significant time, such that we think the current standard does not seem applicable for high-rate, large volume laser uplink technologies. Novel concepts are needed to facilitate efficient utilization of optical uplinks for TC tasking.

\subsection{Impacts on Mission Planning}

To assure solid data throughput between space and ground, the MPS usually relies on stable up- and downlink opportunities within the G/S network of a space mission. Still, within the MPS certain means are commonly present to cope with failed satellite passages. Prioritization of activities is one standard example (cp. the CRIT/DESI concept of the TerraSAR-X/TanDEM-X mission [26], [27], [28], [29]); so called Command Feedback, a confirmation from the TM/TC system to the MPS about the actually initialized SoE, is another option widely used. The issue becomes increasingly problematic the more general availability of a G/S declines. In case of FSO communications external factors like clouds and rain contribute negatively to the station reliability, forcing mission planning to come up with new solutions. 
One might be a reactive planning system, that calculates several possible under- (and over-) performance scenarios in advance. The applicable scenario is then chosen during the pass. As the laser link may not be available (exactly due to bad weather conditions) backup communications to/from the S/C over a separate stable RF connection shall be considered. This might be realized by, e.g. directly from ground by an additional antenna or via global satellite services. Alternatively, an autonomous system on-board the S/C could be employed, judging whether a given pass fulfils the requirements needed.

In the case of an on-board autonomous reaction, it would be mandatory that the S/C manipulates its onboard timeline by itself, e.g. it re-schedules downlinks to other ground stations or it cancels data-takes that are no longer possible due to the lack of free memory at the time of execution. The latter implies larger margins in the model of the memory resource (either in MPS or/and aboard the satellite), dealing with failed passes by reserving alternative ground station contacts to dump data that were not possible to be downloaded in the first place. Reference planning models on ground and on-board the $\mathrm{S} / \mathrm{C}$ are then necessary to assure predictability of the active $\mathrm{SoE}$ at all times and a rapid settlement of the timelines (the one executed by the S/C and the one scheduled by the MPS) is highly desirable.

Regarding the optical uplink of TCs, the challenge is comparable. If the TCs to be sent are not timecritical (e.g. S/W uploads), a failed uplink pass might not have a major impact on the mission planning. Uploads would be re-scheduled to the next possible pass. On the other hand, for time-critical commands the reference model inside MPS relies on a successful uplink of the timeline. Hence, countermeasures have to be implemented to cope with the unreliable uplink channel.

Again, budgeting of larger margins, i.e. spare passes between the first attempts to upload the timeline and the actual start of the on-board SoE, could be one strategy. But this would not allow for a high flexibility, which is needed for short notice high priority data acquisition, e.g. during charter calls. Usage of an OGS network could be another option. This comes with a more demanding interface between control center and ground network, since (1) network connections have to be either changed shortly before or even during the pass, or (2) several connections have to be held open at a time. In any case there needs to be information flow about the actual conditions and a more or less automatic system to react accordingly (also see next section). Further, this reaction has to take place in space as well: The spacecraft's laser terminal needs to point to the factually active ground station, which might be a different one than originally scheduled by MPS for a particular uplink. This station change can either be commanded via a backup RF chain or an on-board autonomy scheme needs to find a stable laser link connection [30].

Finally, the satellite should be able to deal with a complete failure of all measures. Also here, automated on-board planning systems can help to make use of unforeseen free memory aboard the S/C [31], [32].

\subsection{Mitigation of Link Disturbances}

In the system architecture suggested above, failsafe data transmissions between $\mathrm{S} / \mathrm{C}$ and $\mathrm{G} / \mathrm{S}$ are assured by two control loops geared towards disturbances at different time-scales: (1) direct feedback of immediate events (e.g. channel fading) is given from the optical terminal on ground to the space terminal and vice versa by means of ACKs or ARQs; for that the implementation of an optical back-channel is vital; (2) indirect feedback is given to the $\mathrm{S} / \mathrm{C}$, when G/S TM is first processed at the SOC to trigger TCs aboard the $\mathrm{S} / \mathrm{C}$; this can be used to mitigate prospective link failures and might very well not draw on optical uplinks.

ARQ mechanisms may be used to ensure complete fail-safe data transmissions between satellite and ground. It will typically trigger re-transmission of payload data packets, which were lost along the downlink path. This ensures that all data queued for transmission at the space terminal, will be reliably transferred. Still, ARQ shall also be applied to uplink communications, where it will facilitate dependable TC signaling. In this case, the OLEODL serves as the return-channel. Using unique packet identifiers (e.g. in the form of a counter), lost packets can be recognized and requested via the corresponding optical backchannel. This way, the optical communication system can ensure data integrity by itself. Operators do not necessarily need to rely on further infrastructure. At the CCSDS several FSO standards are currently developed with a focus on the physical and data link layers [33]. Higher layer protocols, where ARQ schemes were to be included, shall be standardized in the future.

A straight forward possibility to close the feedback loop between S/C and SOC can be implemented, when mechanisms of CCSDS Service Management (SM) are used [34]. Respective information to be exchanged may include details on the scheduling and booking of satellite passes above the ground stations. Hence, possible configurations for different weather conditions at several station locations shall be prepared in advance or mechanisms shall be developed to alter an existing configuration with updated information (example of such would be change of the coding format or the data rate). In that scenario, the initially planned SoE is nominally 
executed (according to the configuration, contact time, selected station). In case of change of circumstances (e.g. clouds, rain), the Monitoring Data service of the G/S may provide feedback information to the SOC before the execution of a pass, which in turn triggers the configuration change (data rate or coding scheme) or even changes the supporting OGS to another one with good weather conditions. This needs to happen in coordination with S/C control, as the on-board plan needs to be altered respectively.

The long loop over the SOC using the SM interface is certainly not applicable for instantaneous changes (the domain of ARQ, cf. above). However, it allows for much more complex scenarios, extending to the OGS network or even involving the whole ground segment.

\section{Conclusions}

Where free-space optical transmissions are to be deployed for space-to-ground communications, the exploitation of a laser-based uplink channel could provide unique benefits for spacecraft operations, facilitating rapid software uploads and providing increased access-security to a satellite's on-board system. The optical uplink is inherent in such connections via the unavoidable beacon laser from the ground station. Hence, it can be used without extra physical effort.

Modifications necessary to incorporate laser communications to a typical ground segment seem manageable; however, established space data and system standards need to be augmented to cover the challenges arising from the characteristics of the freespace optical channel. Particularly, the necessity for a cloud-free line of sight puts contingent constraints on the availability of such links. Certain data services currently under development promise well to solve resulting hurdles regarding the mission planning and ground station scheduling. To ensure data integrity automated repeat-requests provide a straight way forward. Still, potential tele-command conflicts need to be mitigated, such that novel, customized communication protocols must be considered.

By implication, laser uplinks seem promising ancillary means for future low Earth orbiting space systems during nominal operations. However, bracing for contingencies, ground control will for a certainty rely on additional radio communications guaranteeing emergency backup and fault diagnosis at all times.

\section{Acknowledgements}

The work performed was done (i.a.) by using data from EUMETSAT's Satellite Application Facility on Climate Monitoring (CM SAF). We thank Juliane von Geisau for her support creating the graphic works.

\section{References}

[1] Airbus Defence and Space, "The SpaceDataHighway is open for traffic," Airbus Defense and Space, 23 November 2016. [Online]. Available: http://www.edrsspacedatahighway.com/news/items/thespacedatahighway-is-open-to-traffic. [Accessed 2 August 2017].

[2] H. Hemmati, "Introduction," in Near-Earth Laser Communications, Boca Raton, Florida: CRC Press, Taylor \& Francis Group, 2009, pp. 2-3.

[3] H. Takenaka, Y. Koyama, M. Akioka, D. Kolev, N. Iwakiri, H. Kunimori, A. Carrasco-Casado, Y. Munemasa, E. Okamoto and M. Toyoshima, "In-orbit verification of small optical transponder (SOTA): evaluation of satellite-toground laser communication links," in Proceedings of SPIE, 2016.

[4] A. Biswas, B. Oaida, K. Andrews, J. Kovalik, M. Abrahamson and M. Wright, "Optical payload for lasercomm science (OPALS) link validation during operations from the ISS," in Free-Space Laser Communication and Atmospheric Propagation XXVII, 2015.

[5] C. Schmidt and C. Fuchs, "The OSIRIS program - First results and Outlook," in IEEE International Conference on Space Optical Systems and Applications (ICSOS), Naha, 2017.

[6] N. Perlot, M. Knapek, D. Giggenbach, J. Horwath, M. Brechtelsbauer, Y. Takayami und T. Jono, „Results of the optical downlink experiment KIODO from OICETS satellite to optical ground station Oberpfaffenhofen (OGS$\mathrm{OP})$,“ in Free-space laser communication technologies XIX and atmospheric propogation of electromagnetic waves, San Jose, CA, 2007.

[7] M. T. Knopp, D. Giggenach, R. Mata Calvo, C. Fuchs, K. Saucke, F. Heine, F. Sellmaier and F. Huber, "Connectivity services based on optical ground-to-space links," Acta Astronautica, vol. 148, pp. 369-375, 22 April 2018.

[8] C. Fuchs, N. Perlot, J. Riedi and J. Perdigues, "Performance Estimation of Optical LEO Downlinks," IEEE Journal on Selected Areas in Communications, vol. 36, pp. 1074-1085, May 2018.

[9] R. G. Marshalek, "Pointing, Acquisition and Tracking," in Near-Earth Laser Communications, Boca Raton, FL, CRC Press, Taylor \& Francis Group, 2009, pp. 59-96.

[10] D. Giggenbach, A. Shrestha, C. Fuchs, C. Schmidt and F. Moll, "System Aspects of 
Optical LEO-to-Ground Links," in Proceedings of ICSO, International Conference on Space Optics, Biarritz, 2016.

[11] C. Fuchs and F. Moll, "Ground Station Network Optimization for Space-to-Ground Optical Communication Links," J. Opt. Commun. Netw., vol. 7, pp. 1148-1159, 2015.

[12] F. Moll, "Experimental analysis of channel coherence time and fading behaviour in the LEO-ground link," in IEEE International Conference on Space Optical Systems and Applications (ICSOS), Kobe, Japan, 2014.

[13] L. C. Andrews, R. L. Phillips and C. Y. Hopen, "Aperture Averaging of optical scintillations: power fluctuations and the temporal spectrum," Waves Random Media, vol. 10, pp. 53-70, 2000.

[14] D. Giggenbach and H. Henniger, "Fading-loss assessment in atmospheric free-space optical communication links with on-off-keying," Optical Engineering, vol. 47, 2008.

[15] K.-G. Karlsson, K. Anttila, J. Trentmann, M. Stengel, J. F. Meirink, A. Devasthale, T. Hanschmann, S. Kothe, E. Jääskeläinen, J. Sedlar, N. Benas, G.-J. van Zadelhoff, C. Schlundt, D. Stein, S. Finkensieper, N. Håkansson, R. Hollmann, P. Fuchs and M. Werscheck, "CLARA-A2: CM SAF cLoud, Albedo and surface RAdiation dataset from AVHRR data - Edition 2," Satellite Application Facility on Climate Monitoring, DOI:10.5676/EUM_SAF_CM/CLARA_AVHR R/V002, 2017.

[16] C. Fuchs, S. Poulenard, N. Perlot, J. Riedi und J. Perdigues, „Optimization and throughput estimation of optical ground Networks for LEOdownlinks, GEO-feeder links and GEO-relays,“ in Proceedings of SPIE, 10096. Photonics West, San Francisco, CA, 2017.

[17] CCSDS 911.1-B-4, "Space Link Extension Return All Frames Service Specification," Blue Book (Issue 4), August 2016.

[18] CCSDS 911.2-B-3, "Space Link Extension Return Channel Frames Service Specification," Blue Book (Issue 3), August 2016.

[19] CCSDS 912.1-B-4, "Space Link Extension Forward CLTU Service Specification," Blue Book (Issue 4), August 2016.

[20] CCSDS 921.1-B-1, "Cross Support Transfer Service - Specification Framework," Blue Book (Issue 1), April 2017.

[21] CCSDS 922.1-B-1, "Cross Support Transfer Services - Monitored Data Service," Blue Book (Issue 1), April 2017.
[22] CCSDS 727.0-B-4, "CCSDS File Delivery Protocol (CFDP)," Blue Book (Issue 4), January 2007.

[23] CCSDS 133.1-B-2, "Encapsulation Service," Blue Book (Issue 2), October 2009.

[24] CCSDS 702.1-B-1, "IP over CCSDS Space Links," Blue Book (Issue 1), September 2012.

[25] CCSDS 734.2-B-1, "CCSDS Bundle Protocol Specification," Blue Book (Issue 1), September 2015.

[26] F. Mrowka, R. Metzig, B. Schättler, R. Kahle, C. Lenzen and R. Reissig, "Automation challenges of the Mission Planning System and the Ground Station Network and their Interoperability within the combined TerraSARX/TanDEM-X Ground Segment," in European Ground System Architecture Workshop (ESAW), Darmstadt, 2015.

[27] F. Mrowka, M. P. Geyer, C. Lenzen, A. Spörl, T. Göttfert, E. Maurer, M. Wickler and B. Schättler, "The Joint TerraSAR-X / TanDEM-X Mission Planning System," in Symposium Proceedings IGARSS, Vancouver, Canada, 2011.

[28] C. Lenzen, M. T. Wörle, F. Mrowka, M. P. Geyer and R. Klaehn, "Automated Scheduling for TerraSAR-X/TanDEM-X," in IWPSS, Darmstadt, Germany, 2011.

[29] E. Maurer, F. Mrowka, A. Braun, M. P. Geyer, C. Lenzen, Y. Wasser and M. Wickler, "TerraSAR-X Mission Planning System: Automated Command Generation for Spacecraft Operations," IEEE Transactions on Geoscience and Remote Sensing, vol. 48, pp. 642-648, 2010.

[30] T. Göttfert, C. Lenzen, M. T. Wörle, F. Mrowka and M. Wickler, "Robust Commanding," in 13th International Conference on Space Operations, Pasadena, CA, 2014.

[31] C. Lenzen, M. T. Wörle, T. Göttfert, F. Mrowka and M. Wickler, "Onboard Planning and Scheduling Autonomy within the Scope of the FireBird Mission," in 13th International Conference on Space Operations, Pasadena, CA, 2014.

[32] B. Wille, M. T. Wörle and C. Lenzen, "VAMOS - Verification of Autonomous Mission Planning On-board a Spacecraft," in 19th IFAC

Symposium on Automatic Control in Aerospace, Würzburg, Germany, 2013.

[33] B. L. Edwards, K.-J. Schulz, J. Hamkins, B. Robinson, R. Alliss, R. Daddato, C. Schmidt, D. Giggenbach and L. Braatz, "An Update on the CCSDS Optical Communications Working 
$69^{\text {th }}$ International Astronautical Congress (IAC), Bremen, Germany, 1-5 October 2018.

Copyright (2018 by the International Astronautical Federation (IAF). All rights reserved.

Group," in IEEE International Conference on

Space Optical Systems and Applications,

Okinawa, Japan, 2017.

[34] CCSDS 902.0-G-1, "Extensible Space

Communication Cross Support - Service

Management - Concept," Green Book (Issue 1),

September 2014. 